\section{A RELATIVIZAÇ̃̃O DA COISA JULGADA TRABALHISTA: ABORDAGEM CRÍTICA}

THE RELAXATION OF THE JOB JOINT JUDGMENT: CRITICAL APPROACH LA RELATIVIZACIÓN DE LA COSA JUZGADA: ENFOQUE CRíTICO

Mércia Pereira José Araújo Avelino

- 73 - 84 .
REFLEXÕES ACERCA DO CONTROLE JUDICIAL DA PREVIDÊNCIA SOCIAL

REFLECTIONS ON THE JUDICIAL CONTROL OF SOCIAL SECURITY REFLEXIONES SOBRE EL CONTROL JUDICIAL DE SEGURIDAD SOCIAL

Renato Santiago Quinta Robson Augusto Dainez Condé Jorge Luiz Tesch Santos Marcos dos Santos

. 85 - 96 .

LINHA EDITORIAL E REGRAS DE SUBMISSÃO

EDITORIAL LINE AND SUBMISSION RULES EDITORIAL Y NORMAS DE PRESENTACIÓN DE LA LÍNEA

- 97 - 108 • 